\title{
Learning from Mistakes: Femoral Vein Cannulation-Need for Basic Clinical Guidelines
}

\author{
Nagarajan Muthialu
}

Received: 15 November 2011 / Accepted: 7 March 2012 / Published online: 17 March 2012

(C) Association of Surgeons of India 2012

\section{Dear Editor,}

I read the article by Malviya et al. on "Learning from Mistakes: Femoral Vein Cannulation-An Unusual Complication or a Blessing in Disguise!!!” with interest. It is very interesting and unusual that a rent in diaphragm is diagnosed in this way by computerized tomography done nearly 10 days following the initial traumatic event [1]. What is more interesting is the absence of any clinical findings. Apparently, the scan has also shown about fractured ribs, subcutaneous emphysema, and pneumomediastinum, in addition to collection of blood in intrapleural and retroperitoneal spaces. Basic clinical examination yields more positive clinical findings in such a trauma victim, and a chest X-ray would also have shown the presence of pneumomediastinum.

Continuous requirement of on going transfusions call for urgent evaluation of intrathoracic and intra-abdominal contents by imaging earlier than later. It is indeed a huge volume replacement of 17 units of blood and 25 liters of fluid; otherwise in a trauma victim that makes one wonder whether the imaging should have been done earlier!

Incidentally, a femoral venous cannulation is undertaken in a trauma victim only when the victim is stable and one can safely rule out a vascular injury in thoracoabdominal area. One wouldn't want to transfuse all the blood products into empty spaces in intra-thoracic/abdominal or retroperitoneal spaces as has happened in this situation. Moreover, imaging guidance is now well established and part of routine guidelines to insert any vascular catheter, and is a bedside maneuver. A retrograde Seldinger technique under ultrasound guidance would lessen the chance of such a vascular injury and related complications.

\section{Reference}

1. Malviya A, Yadav JK, Negi N, Gautam Singh CG (2011) Learning from mistakes: femoral vein cannulation - an unusual complication or a blessing in disguise!!! Ind J Surg73:online first

N. Muthialu $(\bowtie)$

Department of Cardiothoracic Surgery,

Great Ormond Street Hospital,

Great Ormond Street,

London WC1N 3JH, UK

e-mail: prathi_naga@hotmail.com 